\title{
Technical Advances to Accelerate Modular Type I Polyketide Synthase Engineering towards a Retro-biosynthetic Platform
}

Pang, Bo; Valencia, Luis E.; Wang, Jessica; Wan, Yao; Lal, Ravi; Zargar, Amin; Keasling, Jay D.

Published in:

Biotechnology and Bioprocess Engineering

Link to article, DOI:

10.1007/s12257-019-0083-9

Publication date:

2019

Document Version

Peer reviewed version

Link back to DTU Orbit

Citation (APA):

Pang, B., Valencia, L. E., Wang, J., Wan, Y., Lal, R., Zargar, A., \& Keasling, J. D. (2019). Technical Advances to Accelerate Modular Type I Polyketide Synthase Engineering towards a Retro-biosynthetic Platform.

Biotechnology and Bioprocess Engineering, 24(3), 413-423. https://doi.org/10.1007/s12257-019-0083-9

\section{General rights}

Copyright and moral rights for the publications made accessible in the public portal are retained by the authors and/or other copyright owners and it is a condition of accessing publications that users recognise and abide by the legal requirements associated with these rights.

- Users may download and print one copy of any publication from the public portal for the purpose of private study or research.

- You may not further distribute the material or use it for any profit-making activity or commercial gain

- You may freely distribute the URL identifying the publication in the public portal 


\section{Lawrence Berkeley National Laboratory}

\section{Recent Work}

\section{Title}

Technical Advances to Accelerate Modular Type I Polyketide Synthase Engineering towards a Retro-biosynthetic Platform

Permalink

https://escholarship.org/uc/item/9nq6g6dz

Journal

Biotechnology and Bioprocess Engineering, 24(3)

\section{ISSN}

1226-8372

\section{Authors}

Pang, B

Valencia, LE

Wang, J

et al.

\section{Publication Date}

2019-06-01

\section{DOI}

10.1007/s12257-019-0083-9

Peer reviewed 


\section{Technical advances to accelerate modular type I polyketide synthase}

\section{2 engineering towards a retro-biosynthetic platform}

3

4

5 Bo Pang ${ }^{\# 1,2,3}$, Luis E. Valencia ${ }^{\# 1,3,4}$, Jessica Wang ${ }^{1}$, Yao Wan ${ }^{1,2,3}$, Ravi Lal ${ }^{1}$, Amin Zargar ${ }^{1,3}$, Jay

D. Keasling $1,2,3,4,5,6,7 *$

7

$8 \quad{ }^{1}$ Joint BioEnergy Institute, Emeryville, CA 94608, USA

$9 \quad{ }^{2}$ QB3 Institute, University of California, Berkeley, CA 94720, USA

$10{ }^{3}$ Biological Systems and Engineering Division, Lawrence Berkeley National Laboratory, Berkeley,

11 CA 94720, USA

$12{ }^{4}$ Department of Bioengineering, University of California, Berkeley, CA 94720, USA

$13{ }^{5}$ Department of Chemical \& Biomolecular Engineering, University of California, Berkeley, Berkeley,

14 CA 94720, USA

$15{ }^{6}$ Novo Nordisk Foundation Center for Biosustainability, Technical University Denmark, DK2970-

16 Horsholm, Denmark

$17{ }^{7}$ Synthetic Biochemistry Center, Institute for Synthetic Biology, Shenzhen Institutes for Advanced

18 Technologies, Shenzhen, Guangdong 518055, China

19

20

$21 *$ Correspondence: Dr. Jay D. Keasling, Joint BioEnergy Institute, 5885 Hollis St. 4th floor,

22 Emeryville, CA 94608, USA; Phone: 510-486-2630, E-mail: jdkeasling@,lbl.gov

23

$24 \quad$ Equal contribution. 


\section{Abstract}

26 Modular type I polyketide synthases (PKSs) are multifunctional proteins that are comprised of 27 individual domains organized into modules. These modules act together to assemble complex 28 polyketides from acyl-CoA substrates in a linear fashion. This assembly-line enzymology makes 29 engineered PKSs a potential retro-biosynthetic platform to produce fuels, commodity chemicals, 30 speciality chemicals, and pharmaceuticals in various host microorganisms, including bacteria and fungi.

31 However, the realization of this potential is restricted by practical difficulties in strain engineering, 32 protein overexpression, and titer/yield optimization. These challenges are becoming more possible to 33 overcome due to technical advances in PKS design, engineered heterologous hosts, DNA synthesis and 34 assembly, PKS heterologous expression, and analytical methodology. In this review, we highlight these 35 technical advances in PKS engineering and provide practical considerations thereof.

\section{Keywords}

37 polyketide synthase, retro-biosynthetic analysis, protein engineering, heterologous expression, 38 biofuels, bioproducts 
Retrobiosynthesis, an approach to design de novo biosynthetic pathways, has enabled the

41 production of various useful chemicals, including biofuels [1] and drugs [2]. In this approach, design

42 starts from the target molecule and proceeds backwards to precursors by considering stepwise

43 biochemical reactions. Modular type I polyketide synthases (PKSs) have the potential to be a versatile

44 retro-biosynthetic platform for its collinear and modular biosynthetic logic. Native PKSs catalyze the formation of carbon-carbon bonds in an assembly-line manner to synthesize the scaffolds of complex natural products, such as macrolides (e.g. 6-deoxyerythronolide B) [3], polyenes (e.g. $\alpha$-lipomycin) [4], and polyethers (e.g. salinomycin) [5]. In this biosynthetic process, each module of a PKS catalyzes a two-carbon elongation followed by potential reductions. During elongation, the acyltransferase (AT) domain loads a specific malonyl-CoA analog onto the phosphopantetheinyl group of the acyl-carrier protein (ACP) domain. This phosphopantetheinyl group is derived from the essential post modification of PKS via phosphopantetheinyl transferases (PPtases). After loading, the ketosynthase (KS) domain, which is primed with an acyl group (a starter acyl group or an acyl chain formed in the previous module), catalyzes a Claisen condensation to fuse the acyl group with the decarboxylated malonyl unit. After elongation, if present, the ketoreductase (KR) domain stereoselectivity reduces the $\beta$-ketone to a hydroxyl group. The dehydratase (DH) domain can then catalyze a dehydration, resulting in the loss of this hydroxyl group to form an $\alpha-\beta$ double bond. Finally, the enoylreductase (ER) domain can reduce this $\alpha-\beta$ double bond to form a saturated bond. The oxidation state of the $\beta$-carbon depends on the presence of reductive domains in each module. Following rounds of elongation and reduction, the acylchain is released by a thioesterase (TE) domain by cyclization to form a macrolactone or hydrolysis to form a linear product. The collinearity of the PKS biosynthetic process results in a diversity of products due to different AT substrate specificities and varying degrees of reductions in each module, and the different module organizations (Fig. 1).

63

Many fundamental studies used engineered PKSs as a retro-biosynthetic platform by conducting domain modifications, domain swaps, and module rearrangements; these results have been 
extensively reviewed elsewhere [6-9]. These studies indicated that there is still a large gap between the

67 promise of PKS engineering and reality, especially for constructing novel multi-module PKSs. Hence, compared to complex natural products, commodity chemicals or biofuels bearing simple structures are more realistic targets for PKS engineering [8]. The production of these targets only requires connecting a limited number of PKS modules (usually $\leq 2$ ). However, even for these simple targets, unique challenges emerge when engineering PKSs to produce compounds in vivo. Besides the fundamentally mechanistic understanding of PKS biosynthesis, these challenges stem from the practical difficulties in novel PKS design, host selection, large DNA fragment synthesis and assembly, target production, and product detection (Fig. 1). In this review, we highlight the state-of-the-art technical advances that deal with these challenges and provide practical considerations for PKS engineering.

\section{PKS design}

To determine a target molecule for production using PKSs, both economic and technical considerations must be evaluated. First, the candidate should be assessed for cost and energy efficiency. Next, retro-biosynthetic analysis can be used to evaluate the feasibility for the candidate molecule to be made through PKS mechanisms. Once a candidate is established to be a feasible target molecule, an initial biosynthetic pathway may be designed using software tools (Fig. 2).

Deciding on a target molecule depends on economic feasibility, which reduces to evaluating the value of the candidate against the cost of its production. An estimate of the candidate's monetary value can be established based on existing commercial value, if known. Otherwise, the value should be estimated according to the candidate's potential applications or molecules with similar functions [10].

To determine production cost, a techno-economic analysis that considers molecular precursors, energy requirements, and production time may be performed [11]. Finally, it is important to consider if the target molecule can be produced in a more practical or economically favorable means through other biosynthetic pathways. While PKSs have recently been engineered to produce short-chain ketones, potential gasoline blending agents, from plant biomass at titers of $1 \mathrm{~g} / \mathrm{L}$ [12], fatty acid and isoprenoid 
synthesis are currently more established production pathways with higher yields [13]. This holistic economic analysis of the target molecule can guide the extent of engineering efforts required for production.

Once established as economically feasible, a retro-biosynthetic analysis can determine a possible pathway for attaining the compound. In this process, the candidate should be deconstructed by carbon-carbon bond breaking according to PKS biosynthetic logic. Engineered PKSs modular nature can be leveraged to programmatically produce non-natural compounds. As such, extensive research has been performed to understand the rules concerning domain swapping to control chain extender unit selection at the acyltransferase (AT) domain and the degree of $\beta$-carbonyl reduction by the ketoreductase (KR), dehydratase (DH), and enoyl reductase (ER) [14-16]. For example, the swapping of non-native ATs has been successfully achieved to switch from methyl-malonyl to malonyl extension [17], and research has been conducted to discover AT's that accept extender units with exotic sidechains (phenyl, allyl, etc.) $[18,19]$. The principles of domain swapping in PKSs are maturing, and there is a wealth of information to determine viability for novel PKS products $[9,20]$. In parallel, considerable progress has been made to engineer PKS domains with more traditional protein engineering methods (e.g. mutagenesis) [21]. With the expanding research on the engineering possibilities of PKSs, the range of molecules that are accessible through PKS biosynthetic pathways is continuously growing.

With a viable target molecule determined, software enables the rapid identification of natural PKS domain candidates to be combined into a suitable catalytic system. Most recently, the free, online software package ClusterCAD [22] has been developed for in silico engineering of PKS pathways to achieve synthesis of a specified product. ClusterCAD semi-quantitatively determines the similarity of the target molecule to the native substrates of an annotated database of biosynthetic gene clusters. Users may additionally specify design constraints, such as native substrate size similarity or AT extender selectivity, to narrow the number of possible routes to the product. The software then proposes biosynthetic pathways that combine components from multiple PKSs that meet the design criteria. ClusterCAD is also just one of the many available in silico tools that help guide the engineering process. 
120 The identification of biosynthetic loci for every known secondary metabolite class [23], the structure-

121 based sequence analysis of PKSs [24], and a crowd-sourced database of microbial PKS and NRPS gene

122 clusters [25] are all accessible from present software tools. Despite such advances in our understanding

123 of PKS engineering, not all design rules for these enzymatic systems have yet been elucidated. As a

124 result, an experimental, combinatorial approach to produce the output PKS systems is often still the

125 most effective method for successful PKS engineering. 


\section{Host selection}

The growth properties of the host organism are important to take into account when selecting a

128 heterologous host for PKS production. Many natural polyketide producing microbes are not culturable

129 in laboratory conditions [26] or grow very slowly in laboratory conditions as is the case with lichen [27]

130 and thus are not suitable hosts for heterologous expression. Bacteria from the genus Streptomyces are common heterologous hosts for PKSs due to their status as prominent PKS producers but they can frequently present issues for industrial scale-up including slow growth and the tendency to form mycelial clumps [28], the latter of which has been shown in some cases to be important in the activation of biosynthetic gene clusters [29]. Despite that, Streptomyces continue to be one of the most popular industrial heterologous hosts for polyketide products and major efforts have been put in place to engineer strains that do not form clumps and grow quickly [30]. Another growth property of interest includes the carbon and energy sources used by the heterologous host. Heterologous hosts such as Pseudomonas putida which consume a wide variety of lignin compounds as a carbon source [31] or the cyanobacterium Synechococcus elongatus, which can use light as an energy source and $\mathrm{CO}_{2}$ as a carbon source [32] have been adapted for the production of PKSs (Table 1).

The availability of genetic tools for a particular host is of great aid to the task of genetically

143 engineering a host to heterologously produce polyketides. Most common heterologous hosts have well developed methods for transformation, conjugation, or transduction of vectors. Of particular importance for controlling expression levels heterologously is a comprehensive list of compatible promoters and RBSs (Fig. 3(A)). These are well known for classic heterologous hosts like E. coli [33] and there have

147 been successful efforts to develop promoter-RBS libraries for Streptomyces [34]. Ultimately, genomic 148 integration of engineered gene clusters is desired in industrial heterologous host strains as it eliminates 149 the need for selection pressures such as antibiotics and provides a more stable and consistent expression of genes [35]. Tools for genomic integration-such as site-specific integrases, homologous recombination-based integration vectors, and CRISPR-Cas9-are widely available for common hosts 
developed for Streptomyces and other suitable PKS hosts the ability to heterologously produce PKSs

154 will continue to improve.

155

When selecting a host for heterologous expression one must consider the existing metabolic

infrastructure to ensure that the host contains all the precursors and tailoring enzymes needed to produce the desired polyketide. Among the most important things to consider is the expression of a compatible PPTase [39] (Fig. 3(B)). As mentioned above, PPTases are responsible for activating ACPs by modifying the active site serine with a phosphopantetheinyl moiety. To ensure the heterologous host can phosphopantetheinylate the engineered PKS in question it is important to ensure the host expresses an appropriate PPTase. While many natural type I PKS producers such as Streptomyces have native PPTases, many common hosts do not and a PPTase must be heterologously expressed. One of the most common PPTases used in heterologous PKS expression is Sfp from Bacillus subtilis because it is capable of phosphopatetheinylating a wide variety of type I PKS and NRPS carrier proteins [40, 41]. However, Sfp may not be active on certain PKSs [42], thus it is important to check for phosphopantetheinylation via proteomics methods [43]. Another important prerequisite is the availability of precursors such as extender units and starter acyl-CoAs. The most common extender units used by PKSs are malonyl-CoA and methylmalonyl-CoA [44] although there do exist a variety of rare extender units as seen in the biosynthesis of zwittermycin [45], chlorizidine [46], or unnatural extender units [47] that can be incorporated by engineered PKSs (Fig. 3(C)). While all organisms produce malonyl-CoA for fatty acid synthesis the ability to produce methylmalonyl-CoA is absent from many common hosts such as E. coli and $S$. cereviseae. This challenge was overcome in E. coli by the

174 development of the K207-3 strain which has both Sfp and a propionyl-CoA carboxylase present to 175 produce intracellular methylmalonyl-CoA [48]. Ultimately a researcher must determine what precursors and tailoring enzymes are necessary for the production of their desired molecule and must take steps to

177 either engineer the heterologous host to produce these precursors or find a heterologous host that naturally produces these precursors and tailoring enzymes. 
181 is possible for the target molecule to be toxic to the host, therefore it is important to do a growth

182 inhibition assay if a sample of the target molecule is available. Additionally, it is possible for the target molecule to be consumed as a carbon source by the host organism. This can be determined by growing the organism on the target molecule as a sole carbon source if possible. Finally, it is possible for the target molecule to be modified or unstable in the cell and this can be detected by incubating the target molecule in cell lysate before extraction and analysis.

\section{DNA synthesis and assembly}

After the target PKS organization and host are determined during the design stage, the careful consideration of the strategy for DNA synthesis and assembly of PKS genes are needed in the build stage (Fig. 4). To expedite the whole engineering process, automation of the steps in the build stage is preferable, although some challenges exist.

192

PKSs are typically encoded by a series of genes clustered in the genome of the producing organisms [49]. Thus, the cloning and expression of PKSs can be achieved by acquiring the genomic DNA (gDNA) harboring the entire cluster from a gDNA library construction. In addition to the phagemediated homologous recombination-based methods [50], transformation-associated recombination (TAR)-based techniques have been developed to capture large biosynthetic gene clusters from environmental DNA or gDNA samples. These techniques utilize the native in vivo homologous recombination of budding yeast Saccharomyces cerevisiae, which occurs in much higher frequency compared to ligation or non-homologous end joining methods [51]. On the other hand, in vitro homology-based methods such as Gibson assembly and sequence- and ligation-independent cloning (SLIC) methods are advantageous over in vivo methods in the turnaround time, although the construct size is typically smaller. 
If the PKS genes are heterologously expressed and require DNA refactoring like codon optimization, it is most likely that de novo DNA synthesis is needed. Although the trends of price per base for DNA sequencing and column-based oligonucleotide synthesis seem to have reached plateaus in recent years, the cost for gene synthesis continues to decrease [52]. As an alternative to outsourcing DNA synthesis, array-based gene synthesis may be carried in house, followed by error correction and verification [53]. In addition, enzymatic de novo synthesis of oligonucleotides with terminal deoxynucleotidyl transferase (TdT)-deoxynucleotide triphosphates (dNTPs) conjugates may serve as a promising basis for enzymatic oligonucleotide synthesizer [54]. Online tools such as JBEI ICE public registry [55] and SynBioHub [56] may facilitate users to search and share the designs of DNA parts. engineering in combination with various promoters, terminators, ribosome-binding sites (RBSs), linker domains, and other parts. If DNA cloning is high-fidelity, sequencing the individual parts may be avoided to reduce cost and effort and save time. Different parts could then be assembled by several methods including yeast assembly [57], Gibson assembly [58], and Golden Gate assembly [59]. In the case of PKS gene construction, although yeast assembly takes a few days, it has the advantages of generating large final gene constructs and promoting recombinational joining of unrelated DNA fragments with the aid of "stitching oligonucleotides", compared with in vitro methods [60]. The construction of shuttle vectors that allow amplification, replication, and expression in $S$. cerevisiae, $E$. coli and the selected host would greatly facilitate this process, as well as the development of automation method such as high-throughput transformation of S. cerevisiae using liquid handling robots [61].

\section{5. Production and analysis}

The introduction of the assembled PKS genes into the selected host yields the candidate strain, which needs the appropriate conditions to produce the target molecules. After production, analytical methods allow researchers to assess the production of the desired product and/or possible shunt products 
231 to evaluate the performance of the engineered PKS. Finally, troubleshooting and optimization can be

232 facilitated by detection of intermediates and high throughput screens.

233

A major consideration in the production stage is the selection of a growth medium. A wellselected medium facilitates the strain's growth and PKS biosynthesis $[62,63]$ and ultimately increases the chance of detecting the final product [64], considering most engineered PKSs result in a decreased production $[6,8]$. The media preferences for the PKS production vary from host to host. For common heterologous hosts the media selection can be straightforward since the media for these organisms are widely used and commercially available. Additionally, PKS production in these organisms is clear, as the precursor biosynthetic genes and PPtase gene are usually under the control of strong constitutive promoters $[48,65,66]$. Hence, rich media are usually the first choice for these hosts. Examples include LB medium used to produce lactones [67, 68] and short chain ketones [17] by engineered PKSs in $E$. coli, while YPD medium was used to produce lactones by engineered PKSs in Saccharomyces cerevisiae [65]. However, as most native PKS producers are not model organisms, the media selection could be challenging when these organisms are chosen as hosts. Rich media usually suitable for cell growth may not be suitable for PKS production in these hosts, since these hosts typically produce their native PKSs under nutritional deficiencies [69]. Therefore, for the PKS production in these non-model hosts, production mediums screens are usually required to produce the desired molecules $[64,70]$ (Table 1).

Once production of the target molecule is confirmed, media optimization is required to maximize the yield and reduce cost. This optimization is crucial for low-value commodity chemicals/biofuels production. In this process, carbon source, nitrogen source, phosphate, and other nutrients are carefully adjusted via a one-factor-at-a-time method or statistical method [71]. In the case of short chain ketone production by an engineered PKS in S. albus, when the carbon source was supplemented by the plant-biomass hydrolysates the product titer increased more than four-fold and further titer increases were achieved by feeding specific acyl-CoA precursors [12]. Aside from 
traditional methods, recently developed machine learning algorithms have great potential to accelerate the media optimization process [72].

Researchers receive feedback regarding the functionality of their engineered PKS when analytical methods are used to detect reaction products. Gas/liquid chromatography coupled to mass spectrometry (GC/LC-MS)-based methods are usually the first choice for analysis $[12,73,74]$ because of their low detection limit for commodity chemicals and biofuels. However, these methods only detect released molecules and leave the PKS biosynthetic pathway a black box. Engineered PKSs frequently fail to produce any free products in vivo in the first Design-Build-Test-Learn (DBTL) cycle. Thus, other analytic approaches are needed to debug these failed PKSs for the next DBTL cycle. Transcriptome [75] and proteomics [76] analysis are well developed to detect the expression of PKSs. Ppant ejection methods are designed to detect on-line PKS intermediates [43]; this method was used to optimize an adipic acid producing PKS in vitro [74] (Fig. 5(B)). Together, these analytical methods can be used effectively to detect the production of target molecules or help troubleshoot malfunctioning modules. route to create a functional PKS in vivo. With well-developed mutagenesis methods, generating a big mutant library is feasible for a specific PKS [77]. The methods used to select or screen for an improved mutant result in the largest bottleneck in a high throughput approach. Screening via GC/LC-MS systems is a possibility [78], however, despite the availability of high throughput GC/LC-MS systems that have reached the speed of several seconds per sample [79], it is still ineffective when the size of mutagenesis library becomes large $\left(>10^{10}\right)$. High throughput detection could be achieved by tying the production of the target molecule with a detectable signal or with the viability of the hosts [80]. For most compounds lacking bioactivity or a chromophore, biosensors are usually required to perform the connections. Although to our knowledge there are no reported cases of a biosensor being coupled with type I PKS production in vivo, some biosensors are reported to detect chemicals that are made or can be possibly made by engineered PKSs, such as diacids [74, 81], lactams [82-84], and pyrones [85, 86]. These compounds are promising targets for high throughput PKS engineering (Fig. 5(B)). 


\section{Conclusions}

288

289

Currently, engineered PKSs can produce several products that are not naturally occurring

290 products in microorganisms. These accomplishments benefit from an expanded understanding of type I modular PKS biosynthetic mechanisms, as well as technical advances summarized here. Similar advances will continue to emerge and promote PKS engineering to be a reliable retro-synthetic platform

293 for the production of commodity chemicals and biofuels. We speculate that over the next few decades,

294 an automatic solution to produce new chemicals by engineered PKS will be available. This technology will include an automatic retro-synthesis software, super hosts for PKS production, automatic DNA synthesis and assembly platforms, and high-throughput production and analytical methods. This has begun to be realized since these technologies have been developed in related fields such as PKS derived natural products discovery [80, 87-89]. By harnessing these advances in PKS engineering and synthetic biology technologies, we will be able to engineer PKS to access an extensive chemical space.

300

301 Acknowledgements

302 This work was part of the DOE Joint BioEnergy Institute (https://www.jbei.org) supported by the U. S.

303 Department of Energy, Office of Science, Office of Biological and Environmental Research. Department of Energy, Energy Efficiency and Renewable Energy, Bioenergy Technologies Office, through contract DE-AC02-05CH11231 between Lawrence Berkeley National Laboratory and the U.

306 S. Department of Energy. This work was also funded by the National Institute of Health 307 (F32GM125179). 


\section{Conflict of interest}

309 J.D.K. has financial interests in Amyris, Lygos, Constructive Biology, Demetrix, Napigen, Maple Bio, 310 and Apertor Labs. 
311 Table 1. Selected cases of engineered PKSs used to produce target chemicals in microbial hosts.

312

\begin{tabular}{|c|c|c|c|c|c|}
\hline Organism & Strain & Media & Product & $\begin{array}{l}\text { Highest } \\
\text { titer }\end{array}$ & Reference \\
\hline \multirow[t]{4}{*}{ Escherichia coli } & K207-3 & $\begin{array}{l}\text { Terrific } \\
\text { Broth (TB) }\end{array}$ & Short-chain ketone & $4 \mathrm{mg} / \mathrm{L}$ & {$[17]$} \\
\hline & K207-3 & $\begin{array}{l}\text { Luria- } \\
\text { Bertani } \\
\text { (LB) }\end{array}$ & Triketide lactone & $23 \mathrm{mg} / \mathrm{L}$ & {$[68]$} \\
\hline & BAP1 & $\mathrm{LB}$ & $\begin{array}{l}\text { 6-deoxyerythronolide } \\
\text { B (6-dEB) analog }\end{array}$ & $1 \mathrm{mg} / \mathrm{L}$ & {$[90]$} \\
\hline & K207-3 & LB & $\begin{array}{l}\text { Long side chain } \\
\text { triketide lactone }\end{array}$ & $14.6 \mathrm{mg} / \mathrm{L}$ & {$[91]$} \\
\hline $\begin{array}{l}\text { Synechococcus } \\
\text { elongatus }\end{array}$ & AMC2302 & BG11 & $\begin{array}{l}\text { Multimethyl- } \\
\text { branched fatty acid }\end{array}$ & $\mathrm{N} / \mathrm{A}^{\mathrm{a}}$ & {$[32]$} \\
\hline $\begin{array}{l}\text { Streptomyces } \\
\text { albus }\end{array}$ & $\mathrm{J} 1074$ & $\begin{array}{l}\text { Modified } \\
042\end{array}$ & Short-chain ketone & $1 \mathrm{~g} / \mathrm{L}$ & {$[12]$} \\
\hline \multirow[t]{2}{*}{$\begin{array}{l}\text { Streptomyces } \\
\text { coelicolor }\end{array}$} & OP & SCFM6-2 & Triketide lactone & $500 \mathrm{mg} / \mathrm{L}^{\mathrm{b}}$ & {$[92]$} \\
\hline & CH999 & R2YE & Triketide lactone & $3 \mathrm{mg} / \mathrm{L}$ & [93] \\
\hline $\begin{array}{l}\text { Streptomyces } \\
\text { lividans }\end{array}$ & $\begin{array}{l}\text { K4.114/ } \\
\text { K4.115 }\end{array}$ & $\mathrm{R} 5$ or R6 & $\begin{array}{l}\text { 6-deoxyerythronolide } \\
\text { B (6-dEB) analog }\end{array}$ & $20 \mathrm{mg} / \mathrm{L}$ & {$[94,95]$} \\
\hline
\end{tabular}




\begin{tabular}{llllll}
\hline $\begin{array}{l}\text { Streptomyces } \\
\text { venezuelae }\end{array}$ & $\begin{array}{l}\text { ATCC } \\
10712\end{array}$ & 042 & $\begin{array}{l}\text { 3-Hydroxycarboxylic } \\
\text { acid }\end{array}$ & $13.8 \mathrm{mg} / \mathrm{L}$ & [64] \\
& ${\mathrm{N} / \mathrm{A}^{\mathrm{a}}}$ & $\mathrm{SCM}$ & $\begin{array}{l}\text { Triketide lactone } \\
\text { N/A }\end{array}$ & [96] \\
& BJ5464 & YPD & Triketide lactone & $1 \mathrm{mg} / \mathrm{L}$ & {$[65]$} \\
$\begin{array}{l}\text { Saccharomyces } \\
\text { cerevisiae }\end{array}$ & & & & & \\
\hline
\end{tabular}

$314{ }^{\text {a }}$ not available

$315{ }^{\mathrm{b}}$ with diketide feeding 
316 Fig. 1. Retro-biosynthetic analysis is a potential platform to bridge native PKSs and engineered PKSs,

317 and the realization of this potential depends on the technical advances in PKS design, host selection,

318 DNA synthesis and assembly, target production, and product analysis. The logic of PKS biosynthesis

319 is illustrated in native PKSs. Acyltransferase (AT) loads the malonyl-CoA analogs (different $\mathrm{R}_{\alpha}$ ) to the

320 acyl carrier protein (ACP). Catalyzed by ketosynthase (KR), the loaded malonyl-ACP decarboxylates

321 and condenses with upstream acyl-ACP intermediate for chain elongation. Optional reduction domains

322 (ketoreductase (KR), dehydratase (DH), and enoylreductase (ER)) reduce the $\beta$-ketone to an alcohol,

323 double bond, or saturated bond $\left(\mathrm{R}_{\beta}\right)$. After rounds of elongation and reductions, the final acyl chain is

324 released by a thioesterase (TE) to form a macrolactone or linear product. Elongation domains are

325 represented as blue sphere, optional reduction domains as green spheres, and release domains as purple

326 spheres.

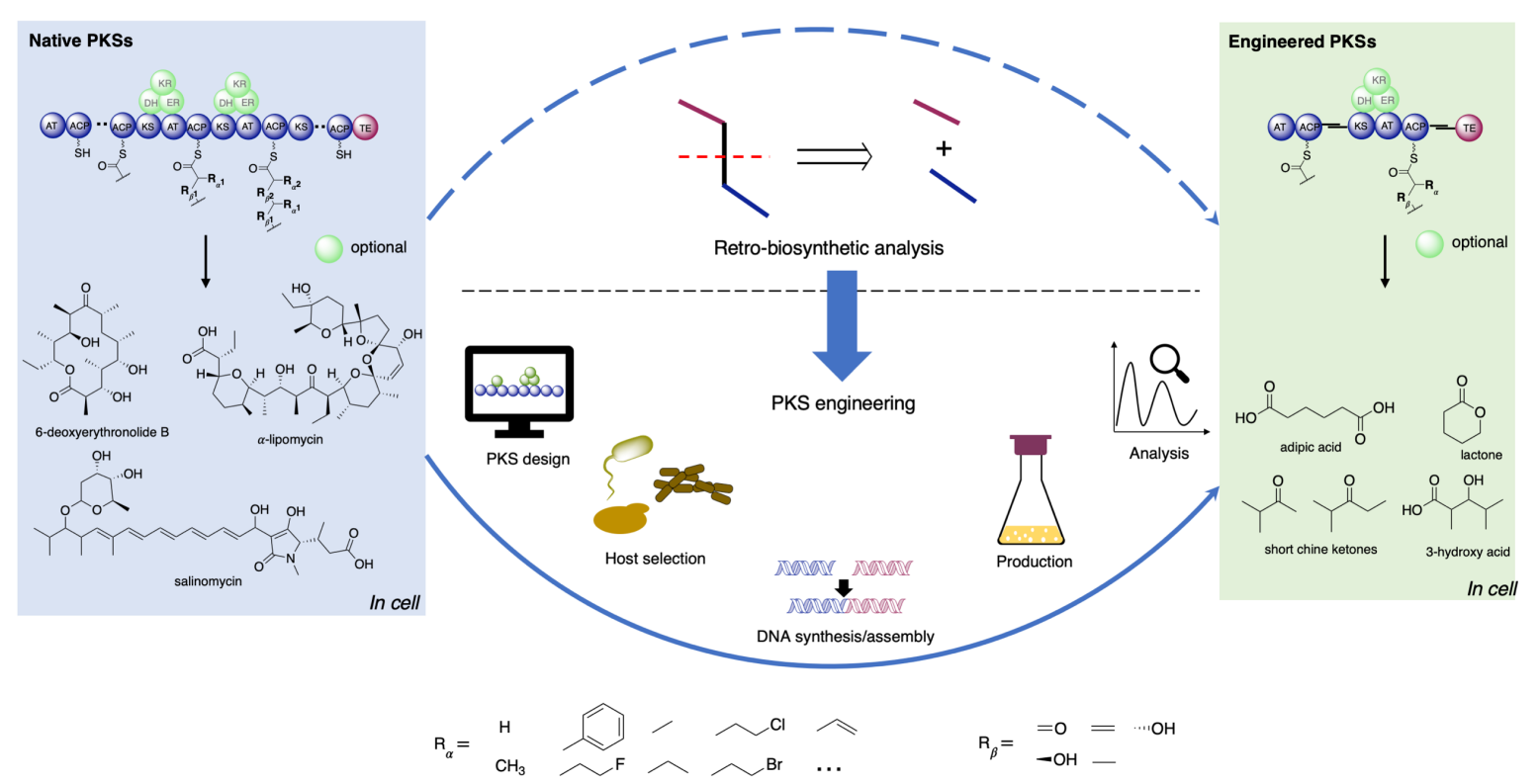


328 Fig. 2. Economic and technical considerations in PKS design

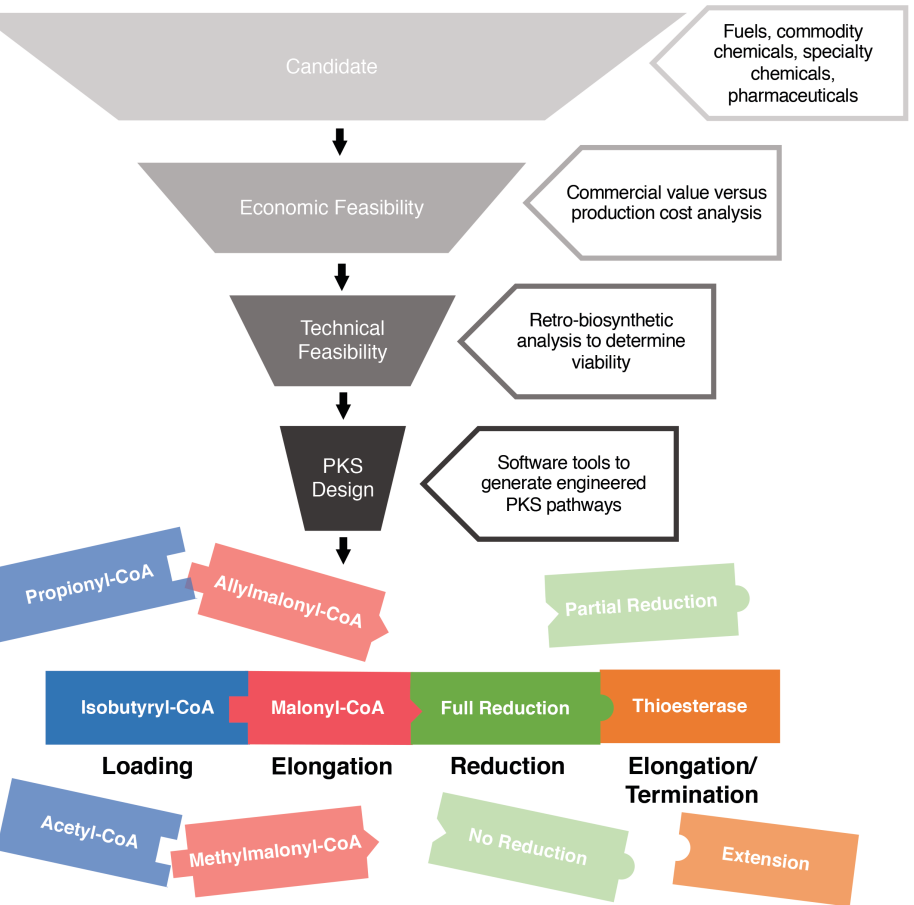


330 Fig. 3. Heterologous host selection must take into account the genetic tools available for the host as

331 well as the metabolic precursors produced by the host. (A) Promoter/RBS libraries help control

332 transcription and translation levels of heterologous proteins. (B) Appropriate PPTases are needed to

333 adequately phosphopantetheinylate heterologous ACP domains. (C) Malonyl-CoA and methylmalonyl-

334 CoA serve as the most common extender unit precursors for type I PKSs though rare extender units

335 exist. RBS - ribosomal binding site, PPTase - phosphopantetheinyl transferase, CoA-SH - coenzyme A,

336 3',5'-PAP - 3',5'-phosphoadenosine phosphate.

337

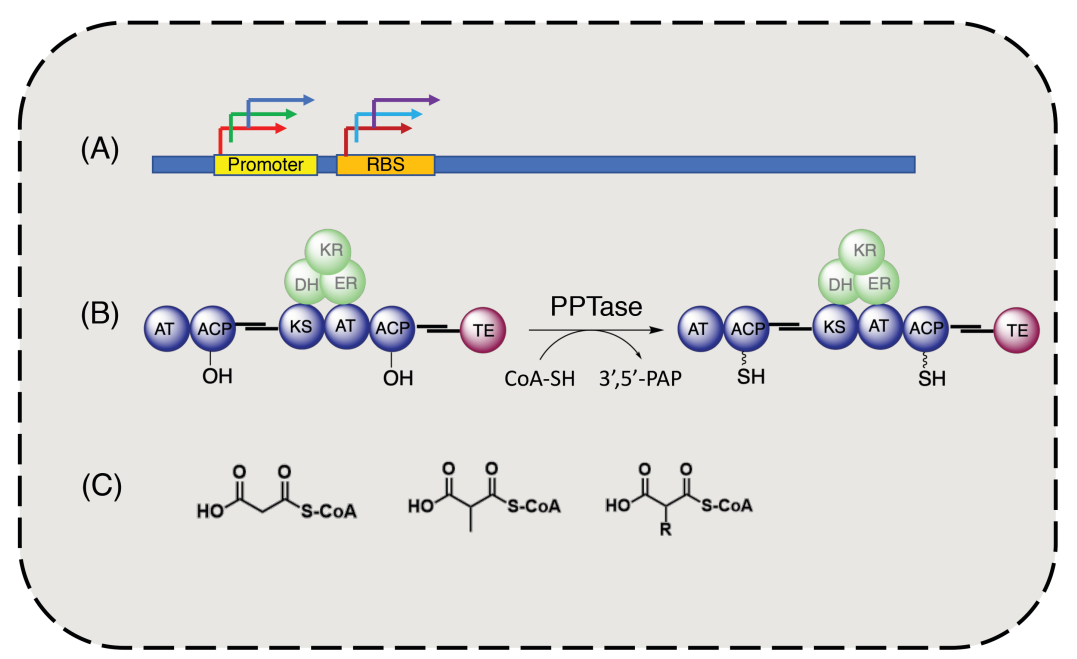


338 Fig. 4. DNA synthesis and assembly in new PKS construction. Purple square represents corresponding

339 homology region for yeast assembly and Gibson assembly, or corresponding sticky end for Golden Gate

340 assembly. gDNA - genomic DNA, RBS - ribosome binding site,

341 Loading/Extension/Reduction/Termination - different modules of PKS genes, Linker - linker domains.

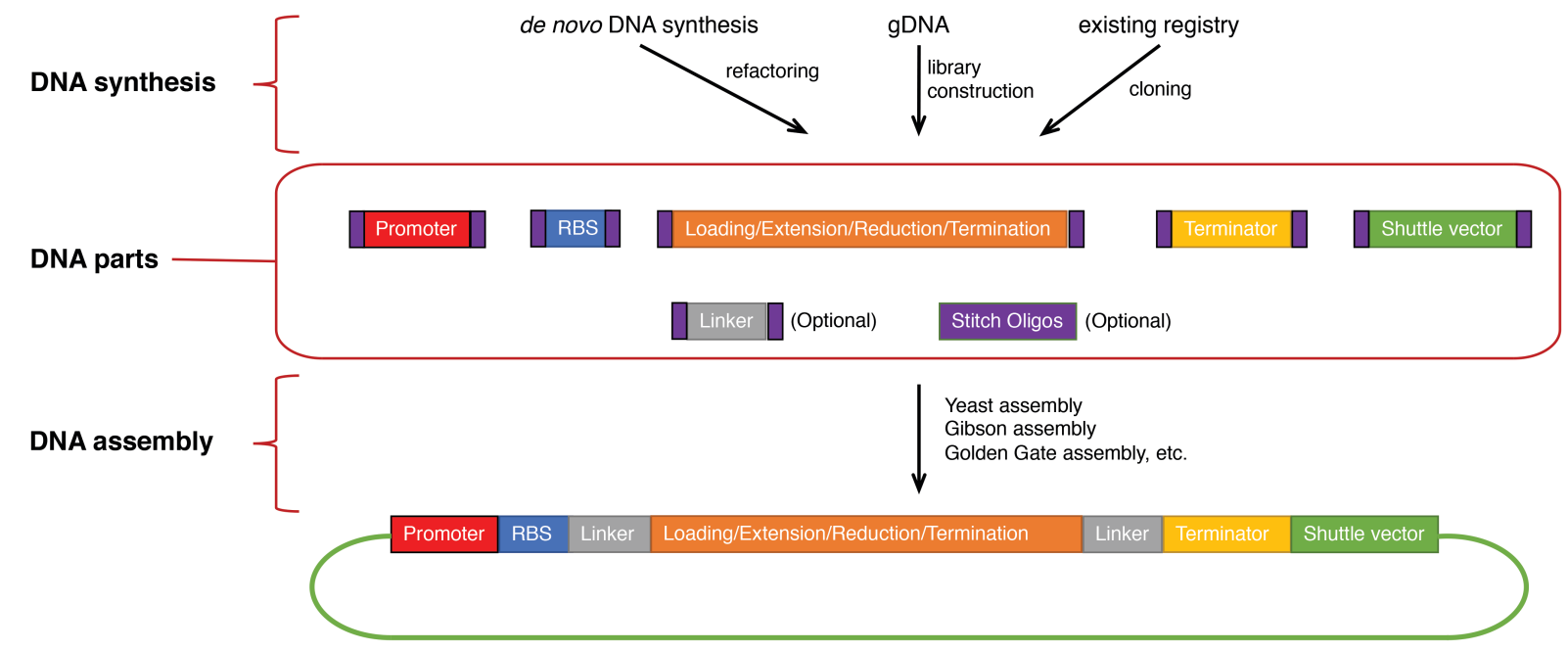


343 Fig. 5. Proposed methods to troubleshoot the stalled PKS in the host: A) Ppant ejection, B) high

344 throughput screen/selection. ESI - electrospray ionization, MS - mass spectrometry, CID - collision-

345 induced dissociation, HCD - higher-energy collisional dissociation, MS/MS - tandem mass

346 spectrometry.

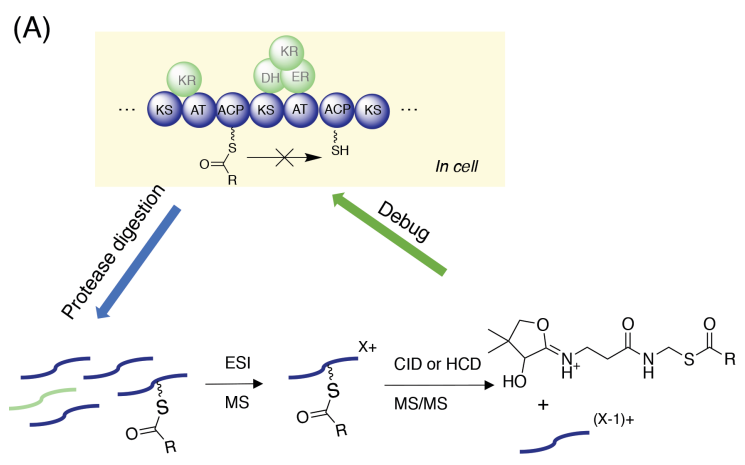

(B)

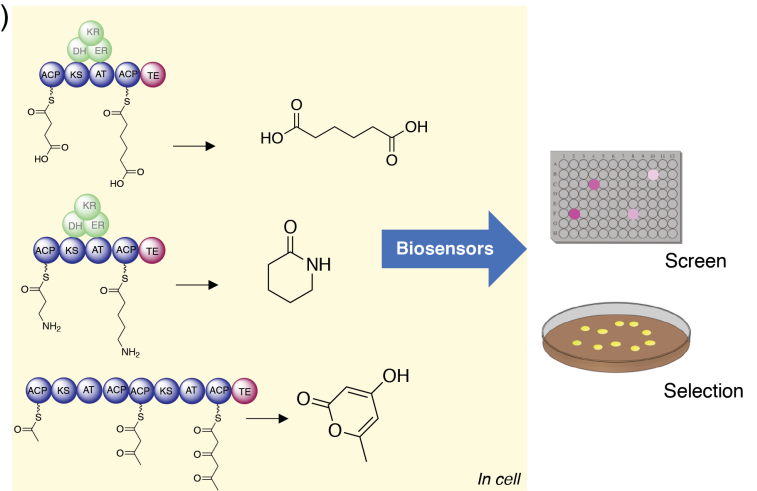




\section{References}

349 1. Sheppard, M.J., A.M. Kunjapur, S.J. Wenck, and K.L.J. Prather (2014) Retro-biosynthetic 350 screening of a modular pathway design achieves selective route for microbial synthesis of 4351 methyl-pentanol. Nat. Commun. 5: 5031.

352 2. Birmingham, W.R., C.A. Starbird, T.D. Panosian, D.P. Nannemann, T.M. Iverson, and B.O. 353 Bachmann (2014) Bioretrosynthetic construction of a didanosine biosynthetic pathway. Nat. Chem. Biol. 10: 392-399.

3. Donadio, S., M.J. Staver, J.B. McAlpine, S.J. Swanson, and L. Katz (1991) Modular organization of genes required for complex polyketide biosynthesis. Science 252: 675-679.

4. Bihlmaier, C., E. Welle, C. Hofmann, K. Welzel, A. Vente, E. Breitling, M. Müller, S. Glaser, and A. Bechthold (2006) Biosynthetic gene cluster for the polyenoyltetramic acid alphalipomycin. Antimicrob. Agents Chemother. 50: 2113-2121.

5. Jiang, C., H. Wang, Q. Kang, J. Liu, and L. Bai (2012) Cloning and characterization of the polyether salinomycin biosynthesis gene cluster of Streptomyces albus XM211. Appl. Environ. Microbiol. 78: 994-1003.

6. Weissman, K.J. (2016) Genetic engineering of modular PKSs: from combinatorial biosynthesis to synthetic biology. Nat. Prod. Rep. 33: 203-230.

7. Barajas, J. F., J. M. Blake-Hedges, C. B. Bailey, S. Curran, and J. D. Keasling (2017) Engineered polyketides: Synergy between protein and host level engineering. Synth. Syst. Biotechnol. 2: 147-166.

8. Yuzawa, S., A. Zargar, B. Pang, L. Katz, and J. D. Keasling (2018) Commodity chemicals from engineered modular type I polyketide synthases. pp. 393-415. In: N. Scrutton (eds.) Methods in

372 9. Klaus, M., and M. Grininger (2018) Engineering strategies for rational polyketide synthase design. Nat. Prod. Rep. 35: 1070-1081.

10. Danzon, P., and A. Epstein (2008) Effects of regulation on drug launch and pricing in interdependent markets, National Bureau of Economic Research, Cambridge, MA. 
11. Davis, R., A. Aden, and P.T. Pienkos (2011) Techno-economic analysis of autotrophic microalgae for fuel production. Appl. Energy 88: 3524-3531.

12. Yuzawa, S., M. Mirsiaghi, R. Jocic, T. Fujii, F. Masson, V.T. Benites, E.E.K. Baidoo, E. Sundstrom, D. Tanjore, T.R. Pray, A. George, R.W. Davis, J.M. Gladden, B.A. Simmons, L. Katz, and J.D. Keasling (2018) Short-chain ketone production by engineered polyketide synthases in Streptomyces albus. Nat. Commun. 9: 4569.

13. Zargar, A., C.B. Bailey, R.W. Haushalter, C.B. Eiben, L. Katz, and J.D. Keasling (2017) Leveraging microbial biosynthetic pathways for the generation of "drop-in" biofuels. Curr. Opin. Biotechnol. 45: 156-163.

14. Keatinge-Clay, A.T., and R.M. Stroud (2006) The structure of a ketoreductase determines the organization of the beta-carbon processing enzymes of modular polyketide synthases. Structure 14: 737-748.

15. Akey, D.L., J.R. Razelun, J. Tehranisa, D.H. Sherman, W.H. Gerwick, and J.L. Smith (2010) Crystal structures of dehydratase domains from the curacin polyketide biosynthetic pathway. Structure 18: 94-105.

16. Zheng, J., D.C. Gay, B. Demeler, M.A. White, and A.T. Keatinge-Clay (2012) Divergence of multimodular polyketide synthases revealed by a didomain structure. Nat. Chem. Biol. 8: 615621.

17. Yuzawa, S., K. Deng, G. Wang, E. E. K. Baidoo, T. R. Northen, P. D. Adams, L. Katz, and J. D. Keasling (2017) Comprehensive in vitro analysis of acyltransferase domain exchanges in modular polyketide synthases and its application for short-chain ketone production. ACS Synth. Biol. 6: 139-147.

18. Mo, S., D.H. Kim, J.H. Lee, J.W. Park, D.B. Basnet, Y.H. Ban, Y.J. Yoo, S. Chen, S.R. Park, E.A. Choi, E. Kim, Y.-Y. Jin, S.-K. Lee, J.Y. Park, Y. Liu, M.O. Lee, K.S. Lee, S.J. Kim, D. Kim, B.C. Park, S. Lee, H.J. Kwon, J.-W. Suh, B.S. Moore, S.-K. Lim, and Y.J. Yoon (2011) Biosynthesis of the allylmalonyl-CoA extender unit for the FK506 polyketide synthase proceeds through a dedicated polyketide synthase and facilitates the mutasynthesis of analogues. J. Am. Chem. Soc. 133: 976-985. 
19. Garcia-Bernardo, J., L. Xiang, H. Hong, B.S. Moore, and P.F. Leadlay (2004) Engineered biosynthesis of phenyl-substituted polyketides. Chembiochem 5: 1129-1131.

20. Zargar, A., J. F. Barajas, R. Lal, and J. D. Keasling (2018) Polyketide synthases as a platform for chemical product design. AIChE J. 64: 4201-4207.

21. Koryakina, I., C. Kasey, J.B. McArthur, A.N. Lowell, J.A. Chemler, S. Li, D.A. Hansen, D.H. Sherman, and G.J. Williams (2017) Inversion of extender unit selectivity in the erythromycin polyketide synthase by acyltransferase domain engineering. ACS Chem. Biol. 12: 114-123.

22. Eng, C.H., T.W.H. Backman, C.B. Bailey, C. Magnan, H. García Martín, L. Katz, P. Baldi, and J.D. Keasling (2018) ClusterCAD: a computational platform for type I modular polyketide synthase design. Nucleic Acids Res. 46: D509-D515.

23. Blin, K., T. Wolf, M.G. Chevrette, X. Lu, C.J. Schwalen, S.A. Kautsar, H.G. Suarez Duran, E.L.C. de Los Santos, H.U. Kim, M. Nave, J.S. Dickschat, D.A. Mitchell, E. Shelest, R. Breitling, E. Takano, S.Y. Lee, T. Weber, and M.H. Medema (2017) antiSMASH 4.0improvements in chemistry prediction and gene cluster boundary identification. Nucleic Acids Res. 45: W36-W41.

24. Khater, S., M. Gupta, P. Agrawal, N. Sain, J. Prava, P. Gupta, M. Grover, N. Kumar, and D. Mohanty (2017) SBSPKSv2: structure-based sequence analysis of polyketide synthases and non-ribosomal peptide synthetases. Nucleic Acids Res. 45: W72-W79.

25. Conway, K.R., and C.N. Boddy (2013) ClusterMine360: a database of microbial PKS/NRPS biosynthesis. Nucleic Acids Res. 41: D402-7.

26. Stewart, E.J. (2012) Growing unculturable bacteria. J. Bacteriol. 194: 4151-4160.

27. Abdel-Hameed, M., R.L. Bertrand, M.D. Piercey-Normore, and J.L. Sorensen (2016) Putative identification of the usnic acid biosynthetic gene cluster by de novo whole-genome sequencing of a lichen-forming fungus. Fungal Biol. 120: 306-316.

28. van Dissel, D., D. Claessen, M. Roth, and G.P. van Wezel (2015) A novel locus for mycelial aggregation forms a gateway to improved Streptomyces cell factories. Microb. Cell Fact. 14: 44.

29. Manteca, Á., and P. Yagüe. (2018) Streptomyces differentiation in liquid cultures as a trigger of 
secondary metabolism. Antibiotics (Basel) 7: 41.

433 30. van Wezel, G.P., P. Krabben, B.A. Traag, B.J.F. Keijser, R. Kerste, E. Vijgenboom, J.J.

$434 \quad$ Heijnen, and B. Kraal (2006) Unlocking Streptomyces spp. for use as sustainable industrial

31. Ravi, K., J. García-Hidalgo, M.F. Gorwa-Grauslund, and G. Lidén (2017) Conversion of lignin model compounds by Pseudomonas putida KT2440 and isolates from compost. Appl. Microbiol. Biotechnol. 101: 5059-5070.

32. Roulet, J., A. Taton, J.W. Golden, A. Arabolaza, M.D. Burkart, and H. Gramajo (2018)

33. Lee, T.S., R.A. Krupa, F. Zhang, M. Hajimorad, W.J. Holtz, N. Prasad, S.K. Lee, and J.D. Keasling (2011) BglBrick vectors and datasheets: A synthetic biology platform for gene

34. Bai, C., Y. Zhang, X. Zhao, Y. Hu, S. Xiang, J. Miao, C. Lou, and L. Zhang (2015) Exploiting

35. Flagfeldt, D.B., V. Siewers, L. Huang, and J. Nielsen (2009) Characterization of chromosomal phosphopantetheinyl transferases: catalysis of a post-translational modification crucial for life. 
Nat Prod Rep 31: 61-108.

461 40. Quadri, L.E., P.H. Weinreb, M. Lei, M.M. Nakano, P. Zuber, and C.T. Walsh (1998)

462

463

464

465

466

467

468

469

470

471

472

473

474

475

476

477

478

479

480

481

482

483

484

485

486

487 Characterization of Sfp, a Bacillus subtilis phosphopantetheinyl transferase for peptidyl carrier protein domains in peptide synthetases. Biochemistry 37: 1585-1595.

41. Kim, J.H., M. Komatsu, K. Shin-Ya, S. Omura, and H. Ikeda (2018) Distribution and functional analysis of the phosphopantetheinyl transferase superfamily in Actinomycetales microorganisms. Proc. Natl. Acad. Sci. USA 115: 6828-6833.

42. Bertrand, R.L., M. Abdel-Hameed, and J.L. Sorensen (2018) Lichen biosynthetic gene clusters. part I. genome sequencing reveals a rich biosynthetic potential. J. Nat. Prod. 81: 723-731.

43. Dorrestein, P.C., S.B. Bumpus, C.T. Calderone, S. Garneau-Tsodikova, Z.D. Aron, P.D. Straight, R. Kolter, C.T. Walsh, and N.L. Kelleher (2006) Facile detection of acyl and peptidyl intermediates on thiotemplate carrier domains via phosphopantetheinyl elimination reactions during tandem mass spectrometry. Biochemistry 45: 12756-12766.

44. Fischbach, M.A., and C.T. Walsh (2006) Assembly-line enzymology for polyketide and nonribosomal Peptide antibiotics: logic, machinery, and mechanisms. Chem. Rev. 106: 34683496.

45. Kevany, B.M., D.A. Rasko, and M.G. Thomas (2009) Characterization of the complete zwittermicin A biosynthesis gene cluster from Bacillus cereus. Appl. Environ. Microbiol. 75: $1144-1155$.

46. Mantovani, S.M., and B.S. Moore (2013) Flavin-linked oxidase catalyzes pyrrolizine formation of dichloropyrrole-containing polyketide extender unit in chlorizidine A. J. Am. Chem. Soc. 135: $18032-18035$.

47. Zhu, X., and W. Zhang (2015) Tagging polyketides/non-ribosomal peptides with a clickable functionality and applications. Front. Chem. 3: 11.

48. Murli, S., J. Kennedy, L.C. Dayem, J.R. Carney, and J.T. Kealey (2003) Metabolic engineering of Escherichia coli for improved 6-deoxyerythronolide B production. J. Ind. Microbiol. Biotechnol. 30: 500-509.

49. Smith, D.J., M.K. Burnham, J.H. Bull, J.E. Hodgson, J.M. Ward, P. Browne, J. Brown, B. 
Barton, A.J. Earl, and G. Turner (1990) Beta-lactam antibiotic biosynthetic genes have been 489 conserved in clusters in prokaryotes and eukaryotes. EMBO J. 9: 741-747.

490 50. Zhang, Y., J.P. Muyrers, G. Testa, and A.F. Stewart (2000) DNA cloning by homologous recombination in Escherichia coli. Nat. Biotechnol. 18: 1314-1317.

492

51. van Leeuwen, J., B. Andrews, C. Boone, and G. Tan (2015) Construction of multifragment plasmids by homologous recombination in yeast. Cold Spring Harb. Protoc. 2015:

494

52. On DNA and Transistors - synthesis. http://www.synthesis.cc/synthesis/2016/03/on_dna_and_transistors.

53. Kosuri, S., and G.M. Church (2014) Large-scale de novo DNA synthesis: technologies and applications. Nat. Methods 11: 499-507.

54. Palluk, S., D.H. Arlow, T. de Rond, S. Barthel, J.S. Kang, R. Bector, H.M. Baghdassarian, A.N. Truong, P.W. Kim, A.K. Singh, N.J. Hillson, and J.D. Keasling (2018) De novo DNA synthesis using polymerase-nucleotide conjugates. Nat. Biotechnol. 36: 645-650.

55. Ham, T.S., Z. Dmytriv, H. Plahar, J. Chen, N.J. Hillson, and J.D. Keasling (2012) Design, implementation and practice of JBEI-ICE: an open source biological part registry platform and

56. McLaughlin, J.A., C.J. Myers, Z. Zundel, G. Misırlı, M. Zhang, I.D. Ofiteru, A. Goñi-Moreno, 506 and A. Wipat (2018) SynBioHub: A standards-enabled design repository for synthetic biology.

57. Gibson, D.G. (2009) Synthesis of DNA fragments in yeast by one-step assembly of overlapping oligonucleotides. Nucleic Acids Res. 37: 6984-6990.

58. Gibson, D.G., L. Young, R.-Y. Chuang, J.C. Venter, C.A. Hutchison, and H.O. Smith (2009) Enzymatic assembly of DNA molecules up to several hundred kilobases. Nat. Methods 6: 343345.

59. Engler, C., R. Kandzia, and S. Marillonnet (2008) A one pot, one step, precision cloning method with high throughput capability. PLoS One 3: e3647.

515 60. DeMarini, D.J., C.L. Creasy, Q. Lu, J. Mao, S.A. Sheardown, G.M. Sathe, and G.P. Livi (2001) 
Oligonucleotide-mediated, PCR-independent cloning by homologous recombination. BioTechniques 30: 520-523.

61. Liu, G., C. Lanham, J.R. Buchan, and M.E. Kaplan (2017) High-throughput transformation of Saccharomyces cerevisiae using liquid handling robots. PLoS One 12: e0174128.

62. Li, C., G. Florova, K. Akopiants, and K.A. Reynolds (2004) Crotonyl-coenzyme A reductase provides methylmalonyl-CoA precursors for monensin biosynthesis by Streptomyces cinnamonensis in an oil-based extended fermentation. Microbiology (Reading, Engl.) 150: $3463-3472$.

63. Kosa, N.M., T.L. Foley, and M.D. Burkart (2014) Fluorescent techniques for discovery and characterization of phosphopantetheinyl transferase inhibitors. J. Antibiot. 67: 113-120.

64. Yuzawa, S., C.B. Bailey, T. Fujii, R. Jocic, J.F. Barajas, V.T. Benites, E.E.K. Baidoo, Y. Chen, C.J. Petzold, L. Katz, and J.D. Keasling (2017) Heterologous gene expression of N-terminally truncated variants of LipPks1 suggests a functionally critical structural motif in the N-terminus of modular polyketide synthase. ACS Chem. Biol. 12: 2725-2729.

65. Mutka, S.C., S.M. Bondi, J.R. Carney, N.A. Da Silva, and J.T. Kealey (2006) Metabolic pathway engineering for complex polyketide biosynthesis in Saccharomyces cerevisiae. FEMS Yeast Res. 6: 40-47.

66. Pfeifer, B.A., S.J. Admiraal, H. Gramajo, D.E. Cane, and C. Khosla (2001) Biosynthesis of complex polyketides in a metabolically engineered strain of E. coli. Science 291: 1790-1792.

67. Watanabe, K., C.C.C. Wang, C.N. Boddy, D.E. Cane, and C. Khosla (2003) Understanding substrate specificity of polyketide synthase modules by generating hybrid multimodular synthases. J. Biol. Chem. 278: 42020-42026.

68. Menzella, H.G., R. Reid, J.R. Carney, S.S. Chandran, S.J. Reisinger, K.G. Patel, D.A. Hopwood, and D.V. Santi (2005) Combinatorial polyketide biosynthesis by de novo design and rearrangement of modular polyketide synthase genes. Nat. Biotechnol. 23: 1171-1176.

69. Bibb, M.J. (2005) Regulation of secondary metabolism in Streptomycetes. Curr. Opin. Microbiol. 8: 208-215.

70. Rateb, M.E., Z. Yu, Y. Yan, D. Yang, T. Huang, S. Vodanovic-Jankovic, M.A. Kron, and B. 
Shen (2014) Medium optimization of Streptomyces sp. 17944 for tirandamycin B production and isolation and structural elucidation of tirandamycins H, I and J. J. Antibiot. 67: 127-132.

546 71. Singh, V., S. Haque, R. Niwas, A. Srivastava, M. Pasupuleti, and C.K.M. Tripathi (2016)

547 Strategies for fermentation medium optimization: An in-depth review. Front. Microbiol. 7:

5482087.

549 72. Costello, Z., and H.G. Martin (2018) A machine learning approach to predict metabolic pathway dynamics from time-series multiomics data. npj Syst. Biol. Appl. 4: 19.

551

552

553

554

555

556

557

558

559

560

561

562

563

564

565

566

567

568

569

570

571

73. Poust, S., A. Hagen, L. Katz, and J.D. Keasling (2014) Narrowing the gap between the promise and reality of polyketide synthases as a synthetic biology platform. Curr. Opin. Biotechnol. 30: $32-39$.

74. Hagen, A., S. Poust, T. de Rond, J.L. Fortman, L. Katz, C.J. Petzold, and J.D. Keasling (2016) Engineering a polyketide synthase for in vitro production of adipic acid. ACS Synth. Biol. 5: $21-27$.

75. Qu, X., C. Lei, and W. Liu (2011) Transcriptome mining of active biosynthetic pathways and their associated products in Streptomyces flaveolus. Angew. Chem. Int. Ed. Engl. 50: 96519654.

76. Bumpus, S.B., B.S. Evans, P.M. Thomas, I. Ntai, and N.L. Kelleher (2009) A proteomics approach to discovering natural products and their biosynthetic pathways. Nat. Biotechnol. 27: 951-956.

77. Packer, M.S., and D.R. Liu (2015) Methods for the directed evolution of proteins. Nat. Rev. Genet. 16: 379-394.

78. Chemler, J.A., A. Tripathi, D.A. Hansen, M. O’Neil-Johnson, R.B. Williams, C. Starks, S.R. Park, and D.H. Sherman (2015) Evolution of efficient modular polyketide synthases by homologous recombination. J. Am. Chem. Soc. 137: 10603-10609.

79. Veach, B.T., T.K. Mudalige, and P. Rye (2017) RapidFire mass spectrometry with enhanced throughput as an alternative to liquid-liquid salt assisted extraction and LC/MS analysis for sulfonamides in honey. Anal. Chem. 89: 3256-3260.

80. Gao, X., X. Xie, I. Pashkov, M.R. Sawaya, J. Laidman, W. Zhang, R. Cacho, T.O. Yeates, and 
Y. Tang (2009) Directed evolution and structural characterization of a simvastatin synthase. Chem. Biol. 16: 1064-1074.

81. Dietrich, J.A., D.L. Shis, A. Alikhani, and J.D. Keasling (2013) Transcription factor-based screens and synthetic selections for microbial small-molecule biosynthesis. ACS Synth. Biol. 2: $47-58$.

82. Barajas, J.F., A. Zargar, B. Pang, V.T. Benites, J. Gin, E.E.K. Baidoo, C.J. Petzold, N.J. Hillson, and J.D. Keasling (2018) Biochemical Characterization of $\beta$-Amino Acid Incorporation in Fluvirucin B2 Biosynthesis. Chembiochem 19: 1391-1395.

83. Zhang, J., J.F. Barajas, M. Burdu, T.L. Ruegg, B. Dias, and J.D. Keasling (2017) Development of a transcription factor-based lactam biosensor. ACS Synth. Biol. 6: 439-445.

84. Yeom, S.-J., M. Kim, K.K. Kwon, Y. Fu, E. Rha, S.-H. Park, H. Lee, H. Kim, D.-H. Lee, D.-M. Kim, and S.-G. Lee (2018) A synthetic microbial biosensor for high-throughput screening of lactam biocatalysts. Nat. Commun. 9: 5053.

85. Tang, S.-Y., S. Qian, O. Akinterinwa, C.S. Frei, J.A. Gredell, and P.C. Cirino (2013) Screening for enhanced triacetic acid lactone production by recombinant Escherichia coli expressing a designed triacetic acid lactone reporter. J. Am. Chem. Soc. 135: 10099-10103.

86. Hughes, A.J., J.F. Detelich, and A.T. Keatinge-Clay (2012) Employing a polyketide synthase module and thioesterase in the semipreparative biocatalysis of diverse triketide pyrones. Medchemcomm 3: 956.

87. Dejong, C.A., G.M. Chen, H. Li, C.W. Johnston, M.R. Edwards, P.N. Rees, M.A. Skinnider, A.L.H. Webster, and N.A. Magarvey (2016) Polyketide and nonribosomal peptide retrobiosynthesis and global gene cluster matching. Nat. Chem. Biol. 12: 1007-1014.

88. Osswald, C., G. Zipf, G. Schmidt, J. Maier, H.S. Bernauer, R. Müller, and S.C. Wenzel (2014) Modular construction of a functional artificial epothilone polyketide pathway. ACS Synth. Biol. 3: 759-772.

89. Myronovskyi, M., B. Rosenkränzer, S. Nadmid, P. Pujic, P. Normand, and A. Luzhetskyy (2018) Generation of a cluster-free Streptomyces albus chassis strains for improved heterologous expression of secondary metabolite clusters. Metab. Eng. 49: 316-324. 
600

601

602

603

604

605

606

607

608

609

610

611

612

613

614 96. Yan, J., C. Hazzard, S.A. Bonnett, and K.A. Reynolds (2012) Functional modular dissection of

90. Lee, H.Y., C.J.B. Harvey, D.E. Cane, and C. Khosla (2011) Improved precursor-directed biosynthesis in E. coli via directed evolution. J. Antibiot. 64: 59-64.

91. Menzella, H.G., J.R. Carney, and D.V. Santi (2007) Rational design and assembly of synthetic trimodular polyketide synthases. Chem. Biol. 14: 143-151.

92. Regentin, R., J. Kennedy, N. Wu, J.R. Carney, P. Licari, J. Galazzo, and R. Desai (2004) Precursor-directed biosynthesis of novel triketide lactones. Biotechnol. Prog. 20: 122-127.

93. Kao, C.M., L. Katz, and C. Khosla (1994) Engineered biosynthesis of a complete macrolactone in a heterologous host. Science 265: 509-512.

94. Xue, Q., G. Ashley, C.R. Hutchinson, and D.V. Santi (1999) A multiplasmid approach to preparing large libraries of polyketides. Proc. Natl. Acad. Sci. USA 96: 11740-11745.

95. Kato, Y., L. Bai, Q. Xue, W.P. Revill, T.-W. Yu, and H.G. Floss (2002) Functional expression of genes involved in the biosynthesis of the novel polyketide chain extension unit, methoxymalonyl-acyl carrier protein, and engineered biosynthesis of 2-desmethyl-2-methoxy6-deoxyerythronolide B. J. Am. Chem. Soc. 124: 5268-5269.

615 DEBS1-TE changes triketide lactone ratios and provides insight into acyl group loading,

616 hydrolysis, and ACP transfer. Biochemistry 51: 9333-9341. 